\title{
Las organizaciones empresariales y las empresas ante la conciliación de la vida laboral y familiar-personal ${ }^{*}$
}

\section{Francesc Gibert}

Andreu Lope

Ramon de Alós

Universitat Autònoma de Barcelona. Departament de Sociologia

Grup d'Estudis Sociològics sobre la Vida Quotidiana i el Treball

08193 Bellaterra (Barcelona). Spain

francesc.gibert@uab.cat

andreu.lope@uab.cat

ramon.dealos@uab.cat

\section{Resumen}

El artículo analiza las iniciativas empresariales españolas más innovadoras en torno a la conciliación de la vida laboral, familiar y personal. A partir del estudio de la posición general del empresariado, se centra específicamente en la de sus organizaciones confederales, de las sectoriales de químicas, alimentación y comercio ante la legislación y la negociación colectiva, así como en el estudio de tres iniciativas de grandes empresas. Con estos datos, el empresariado español es poco innovador, muy condicionado por exigencias legales y próximo al modelo de family-friendly, de corte limitado en sus acciones más significativas.

Palabras clave: empresariado, organizaciones empresariales, políticas de recursos humanos, negociación colectiva y conciliación de la vida laboral, familiar y personal.

\section{Abstract. How employers' organizations and companies deal with work/family/personal life arrangement}

This article analyzes the most innovative Spanish employer initiatives on work/family/personal life arrangement. The research is based on a previous contextualization of Spanish employer to focus specially on confederal employers' organizations and chemical, food and retail significant employers' organizations deal with legislation and collective bargaining, as well as on the initiatives of three big companies. According to these data, the Spanish employer is scarcely innovative, markedly conditioned by legal requirements and closely related to limited versions of family-friendly model in its most significant initiatives.

Key words: employer, employers' organizations, human resource management, collective bargaining and work/family/personal life arrangement.

* Este artículo se basa en la investigación El tiempo de trabajo en la negociación colectiva y sus efectos sociales, financiada por el Ministerio de Ciencia y Tecnología y realizada por el QUIT, los objetivos específicos de la cual se detallan en el artículo de Carrasquer, Martín Artiles y Massó (2006) de este monográfico. 


\section{Sumario}

\section{Introducción}

2. La regulación sobre la conciliación laboral, familiar y personal y la perspectiva de las organizaciones empresariales
3. Las empresas punteras en conciliación

4. Conclusiones

Entrevistas referenciadas

Bibliografía

\section{Introducción}

La legislación española recoge los criterios adoptados en directivas europeas respecto a la conciliación y, en alguna medida, amplía las medidas que en ellas se efectúan con carácter de mínimos. Aún y con esa ampliación, España no está ubicada entre los países que ofrecen mayores facilidades a la conciliación de la vida laboral y familiar y, paralelamente, dedica un esfuerzo económico inferior a la media de la UE-15 para atender a los costes derivados de las licencias parentales.

Si a ello añadimos que, como señala el Consejo Económico y Social (2003), la negociación colectiva ha desempeñado un papel poco relevante, aunque creciente, en este sentido, parece difícil que en las empresas abunden experiencias que fomenten la conciliación de forma decidida. Nuestra investigación preveía la existencia de casos significativos y relevantes en esa dirección, pero, al mismo tiempo, casos que adquieren esa especial significación por su relativa excepcionalidad en el tejido empresarial ${ }^{1}$.

De hecho, la propia idea de conciliación de la vida laboral, familiar y personal ha estado ausente hasta fechas muy recientes en el discurso empresarial en España y no es hasta finales de los noventa que se aprecia una creciente atención a esa temática, por lo que puede afirmarse que hoy no resulta extraña en ámbitos empresariales. Para comprender mejor el significado o los significados que adquiere la idea de conciliación entre el empresariado, resulta conveniente recordar cuáles han sido las principales vías que han motivado su consideración. Éstas son dos. La primera vía ha sido la transposición a la legislación española de las directivas europeas: esta vía adquiere un carácter impuesto y universal, en tanto obliga a las empresas a adaptarse a los supuestos que

1. De los pocos estudios sobre el tema, podemos advertir que Chinchilla y Poelmans (2003), del Centro Internacional de Trabajo y Familia del IESE (Universidad de Navarra), destacan que apenas el $10 \%$ de las empresas españolas tiene un conjunto de políticas familiarmente responsables, mientras que Chinchilla (2004) apunta a un 20\%. Menos optimista es el informe Randstad, del Instituto de Estudios Laborales de ESADE (2004), o el estudio Work and Life Balance, de CREADE (2001), según el cual las empresas españolas se sitúan en el grupo de países que ofrecen menos políticas para facilitar la adaptación de la vida laboral, familiar y personal. En torno a la valoración de empresas y trabajadores, véase GPI Consultores (2005), con datos que validan la excepcionalidad de medidas empresariales proactivas relevantes y significativas. 
prevé y se concreta en la negociación colectiva. La segunda vía, en cambio, es deliberada: la conciliación se contempla en determinados círculos empresariales, en cuanto se considera que ofrece nuevas oportunidades en la gestión de personal.

Las dos vías son de especial importancia para entender las interpretaciones y las prácticas en torno a la conciliación por parte de las empresas. De ella, se extraen los referentes y las estrategias más novedosas y racionalizadas, a menudo, aunque no todas, referenciadas por parte de la literatura managerial como «buenas prácticas». Así, por lo que se refiere a las empresas españolas en general, se entiende que las medidas de apoyo a la conciliación suponen un coste adicional; básicamente, un coste en términos organizativos. Si sus posibles beneficios no alcanzan el montante de los costes, la conciliación sólo suele ser asumida en la medida en que obliga la legislación. De ahí las fuertes tendencias empresariales para que la negociación colectiva no generalice entre las plantillas más allá de las disposiciones en esta materia que establece la legislación y que, por tanto, en pocos convenios asistamos a regulaciones singulares. Pero, como apunta la segunda vía antes mencionada, la conciliación también puede revertir en mayor motivación y compromiso del empleado con la organización y, en consecuencia, en una optimización de su productividad y eficiencia.

En este artículo, trataremos los casos que hemos estudiado a modo de ejemplos singulares, ubicándolos en el marco de las experiencias que adoptan las empresas que desarrollan prácticas vinculadas a la conciliación. Antes de eso, situaremos la posición de las organizaciones empresariales al respecto $y$, para una mejor contextualización de los casos estudiados, analizaremos los convenios colectivos sectoriales en que se ubican las empresas escogidas para dicho estudio. Más concretamente, observaremos la posición de la CEOE ante la conciliación y estudiaremos los casos de tres grandes empresas que etiquetamos como Alimentación-Comercio, Química y Farmacia. A las dos últimas, elaboradoras de productos químicos y farmacéuticos, les atañe el convenio del sector químico, muy significativo por recoger las medidas más innovadoras en materia de conciliación, y a la primera, el del sector de productos dietéticos y preparados alimenticios. Asimismo, la última y la primera son empresas con iniciativas entendidas como de «buenas prácticas» por parte de la literatura managerial española. Analizaremos ambos convenios antes de tratar los casos de las tres empresas, el de química de carácter estatal y el de preparados alimenticios válido para Cataluña, ya que estudiamos el caso de un centro de producción de alimentación-comercio allí ubicado. También analizaremos el convenio catalán del sector de comercio, por regular un tipo de actividades a través de una presencia horaria que interviene de forma manifiesta sobre la conciliación en una doble vía: a) sobre las posibilidades de conciliar la vida laboral y familiar de los empleados del sector, y b) sobre las posibilidades del conjunto de la población de conciliar ambas cuestiones, pudiendo acceder a la compra de productos necesarios para la actuación cotidiana en horarios no ocupados por el desarrollo de la actividad 
laboral. Finalmente, expondremos los argumentos más significativos esgrimidos por los responsables de las organizaciones empresariales catalanas del sector químico y del comercio, expresadas a través de sendas entrevistas en profundidad, con el fin de ampliar la perspectiva sobre la posición de esas organizaciones empresariales sectoriales ${ }^{2}$.

\section{La regulación sobre la conciliación laboral, familiar y personal y la perspectiva de las organizaciones empresariales}

Retomando nuestra argumentación inicial, cabe recordar que las iniciativas comunitarias al respecto de la conciliación, se inician tímidamente con la Recomendación del Consejo Europeo 92/241/CEE de 21 de marzo de 1992, que insta a los estados miembros a adoptar medidas que fomenten el reparto de responsabilidades entre hombres y mujeres en lo que se refiere al cuidado de los hijos. Posteriormente, la Directiva europea 1992/85/ del Consejo efectúa una serie de disposiciones en materia de maternidad y la Directiva 1996/34/CE lo hace sobre los permisos parentales, basándose en el acuerdo marco previo firmado por las organizaciones empresariales europeas UNICE y CEEP, y la Confederación Europea de Sindicatos (European Industrial Relations Observatory, 2002).

Todas esas directivas dotan de una serie de requisitos y criterios mínimos a las políticas de conciliación, que se concretan de forma muy diversa en los estados miembros a través de su legislación y por medio de los avances en ese orden de la negociación colectiva (López y Valiño, 2004). Hay que subrayar, en cualquier caso, la importancia de las directivas comunitarias para impulsar desarrollos legislativos en el tema que nos ocupa en diversos países. Y más todavía cuando, con posterioridad, la Estrategia Europea de Empleo definirá como uno de sus objetivos fundamentales el aumento de la tasa de actividad y de la tasa de empleo de las mujeres, a tono con uno de los cuatro pilares de su política de empleo (el fomento de la igualdad entre hombres y mujeres en el mercado laboral). Entre otras cuestiones de especial relieve vinculadas a las desigualdades de género, las políticas de conciliación de la vida laboral, familiar y personal tendrán como objetivo explícito facilitar la incorporación y el mantenimiento de la mujer en el mercado de trabajo, vinculándose así estrechamente a la antes mencionada Estrategia Europea (Torns, 2005).

En España, las directivas europeas se concretaron en la Ley 39/1999, del 5 de noviembre, de conciliación de la vida laboral y familiar de las personas trabajadoras. Una ley, elaborada desde el gobierno del Partido Popular, que las organizaciones sindicales han tildado de tímida e incompleta, por no incorporar, entre otras cuestiones, una política integral que incluyera aspectos rela-

2. Las entrevistas que basan nuestro análisis, de las cuales indicamos algunas citas significativas, han sido codificadas considerando todas las entrevistas de la amplia investigación en la que se inserta este trabajo (Carrasquer, Martín Artiles y Massó, 2006). Al final del artículo, se indica la codificación de las entrevistas que aquí se referencian. 
tivos al desarrollo de guarderías y residencias y centros de día públicos. Una ley, en cualquier caso, que amplía los derechos promovidos en concepto de mínimos por las directivas europeas.

De hecho, en gran medida, las políticas de conciliación se fundamentan en la regulación de una serie de cuestiones básicas que remiten, sobre todo, a la atención al cuidado de los hijos, aunque, como es sabido, la atención que muchas mujeres prestan a personas mayores es un elemento que dificulta sobremanera - especialmente en España - su actividad laboral. Así, destacan en aquella regulación cuestiones ligadas a: licencias de maternidad; permisos por nacimiento de hijo; permisos y excedencias que tienen que ver con el cuidado de los hijos en edades tempranas, y licencias por motivos familiares urgentes, para atender a familiares en caso de enfermedad o discapacidad (Torns, 2005).

Con todo, España, pese a ampliar las recomendaciones comunitarias, está situada en los puestos menos privilegiados en ese sentido en la UE-15. Así, por ejemplo: las excedencias en el caso español son siempre sin remuneración; en cambio, Suecia, Gran Bretaña, Finlandia o Dinamarca facilitan hasta dos o tres semanas por nacimiento del hijo. El gasto social por prestaciones económicas por licencias parentales se sitúa claramente por debajo de la media de la UE-15 e, igualmente, son diversos los países que limitan menos que la legislación española las licencias por motivos familiares urgentes (CES, 2004).

La situación del gasto público español vinculado al fomento a la conciliación no es, tampoco, positiva en términos comparativos con otros países europeos, sin embargo, en otro orden de cosas, la Administración del Estado ${ }^{3}$ ha iniciado el proceso de promover entre los funcionarios medidas tendentes a hacer compatibles para hombres y mujeres el desarrollo de una carrera profesional con la atención y el cuidado a la familia; aunque, como es conocido, quienes acaban acogiéndose a ese tipo de medidas son casi exclusivamente mujeres ${ }^{4}$. Con ello, afirma querer servir de ejemplo al sector privado y seguir el criterio de la Ley de conciliación, que atribuye al poder público el deber de promoverla. En concreto, y tras aprobarse en Consejo de Ministros del 4 de marzo del 2005, se contempla aplicar una serie de medidas en la

3. La Administración pública de algunas comunidades autónomas, como la de Cataluña entre otras, hace algún tiempo que ha desarrollado, y sigue impulsando, diversas medidas para favorecer las posibilidades de conciliación entre la vida laboral y familiar de sus empleados. Además, dos terceras partes de las comunidades autónomas proporcionan ayudas económicas en función de los hijos (Cataluña desde el primer hijo, Navarra y el País Vasco desde el segundo y Castilla-León desde el tercero), con cuantías, requisitos y períodos variables en cada caso (El País Semanal, 2005).

4. En CES (2004) se recogen datos de un estudio de la Fundación Europea para la Mejora de las Condiciones de Vida y de Trabajo, según el cual el 85\% de los hombres de la UE dicen no haber pedido ni pensar hacerlo licencias parentales, dato que asciende al 95\% de los españoles. De hecho, según esa misma fuente, el 97,9\% de permisos familiares en España en el 2003 han sido disfrutados por mujeres. 
Administración pública, que, previa negociación con los sindicatos, permita impulsar la jornada flexible (modificando, además, la jornada y extendiéndola de las 9 a las 18 horas), así como la jornada reducida por el cuidado de hijos menores, de personas mayores o de discapacitados, entre otras cuestiones (Cuenca, 2005).

Las alusiones de CEOE y CEPYME a la conciliación de la vida laboral, familiar y personal son escasas. En un primer momento, tras la aprobación de la Ley 39/1999, la posición manifestada por ambas organizaciones es la de considerar la conciliación como una obligación externa que amenaza a la contención de costes laborales y, en consecuencia, la competitividad de las empresas. Así, por ejemplo, en la Circular para la negociación colectiva, de 2001, se advierte de los costes para las empresas que puede suponer cualquier acuerdo sobre esta materia (CEOE, 2002). Asimismo, en un artículo reciente, un responsable de la organización empresarial catalana PIMEC, representativa de la pequeña empresa y afiliada a CEPYME, expone que: «No es en la empresa donde se ha resolver todo esto [la conciliación)] ni es la empresa la que tiene que cambiar la cultura de nuestra sociedad. Tampoco quien tiene que plantear que no es razonable ni práctico que todos hagamos los mismos días de fiesta y unos horarios más o menos idénticos. La solución no es regular ni instituir nuevos permisos para seguir complicando la vida dentro de las empresas, sino que es en otros ámbitos y colectivos en los que hay que pensar» (Casals, 2005). No obstante, habitualmente se matiza dicha posición y las organizaciones empresariales confederales abogan, junto con los sindicatos, por un tratamiento de la jornada laboral, vacaciones, programación de la formación, etc., que permita conciliar las necesidades productivas y las de índole personal o familiar, como queda recogido en el Acuerdo interconfederal para la negociación colectiva de 2005 (2005).

Apuntábamos, al inicio, que la negociación colectiva en España no ha proporcionado avances sustanciales con respecto a la legislación en términos de promover la conciliación de la vida laboral, familiar y personal. En este orden de cosas, el balance hecho por la CEOE (2004) de la negociación colectiva en el 2003 indica que un 19\% de los convenios mejora las disposiciones legales sobre licencias y permisos para promover la conciliación, aunque dichas mejoras las más de las veces son modestas (Argüelles, Martínez y Menéndez, 2004; Instituto de Estudios Laborales, 2004). La "cata» que en su momento realizamos en una fase inicial de nuestra investigación, mostraba también esa lenta progresión, sobre todo en términos de inclusión de cláusulas que repiten las disposiciones legislativas. Esa repetición no es ociosa. La legislación es poco conocida por los asalariados y el que un convenio recoja los principales elementos de la Ley de conciliación sirve para aumentar su grado de conocimiento — también entre los empresarios - y, por tanto, para facilitar el uso de los derechos que proporciona. El análisis que desarrollamos a continuación de los convenios de los sectores en que se insertan las empresas que hemos estudiado, añade mayor precisión a las tendencias y cuestiones hasta aquí indicadas. 
El convenio de preparados alimenticios de Cataluña ${ }^{5}$ mejora alguna de las cuestiones establecidas legislativamente en materia de conciliación. Entre las medidas relacionadas, siquiera parcialmente con ella, destacan:

- Jornada: limitación a un máximo de cinco horas la jornada del sábado para el personal administrativo y comercial.

- Permisos: amplía a tres días el permiso retribuido por muerte o enfermedad grave de familiares. Se facilitan cuatro horas de permiso por asuntos propios que se extiende a un día (ocho horas) a partir del 2005. Los permisos por matrimonio pueden vincularse parcialmente al período vacacional y se podrá acompañar, con justificación documental, a los hijos de hasta catorce años a visitas médicas urgentes.

- Situación de riesgo por embarazo: se recoge la obligación de las empresas de complementar las prestaciones económicas de la Seguridad Social con motivo de declaración de incapacidad temporal por riesgo de embarazo.

- Ayudas por hijos o cónyuges disminuidos físicos o psíquicos y ayuda escolar: se establecen ayudas por ambos motivos, siendo sensiblemente más reducidas las ayudas escolares para hijos de entre seis y dieciséis años.

Por su parte, el convenio del comercio ${ }^{6}$ es menos beneficioso para los asalariados. Se ajusta a la legislación y reproduce parte de los derechos que la misma establece en materias como: licencias por nacimiento o por enfermedad grave de familiares (en este caso, la licencia se extiende hasta tres días); licencias por lactancia; reserva de puesto de trabajo por excedencia de maternidad; etc. Sin embargo, se fijan severas limitaciones al ejercicio de esa excedencia, mientras que, por otro lado, se concede un día de permiso para asuntos propios.

Finalmente, el convenio del sector químico ${ }^{7}$ se reconoce habitualmente como uno de los más avanzados, en el sentido de que, desde hace años, en sus cláusulas se recogen temas que después son abordados en otros convenios y se recogen, además, de forma notoriamente más amplia que en otros sectores. A tenor de esa tradición, visible en convenios anteriores en aspectos relativos al empleo o a la formación, el convenio de químicas muestra una presencia mucho mayor que los anteriormente tratados (y, en general, que en el conjunto de convenios colectivos sectoriales) sobre aspectos vinculados a la conciliación.

5. El convenio del sector de productos dietéticos y preparados alimenticios para los años 20032004, en los que se desarrolla nuestra investigación, es firmado por las federaciones catalanas implicadas de los sindicatos UGT y CCOO y por la Asociación Española de Fabricantes de Preparados Alimenticios Especiales, Dietéticos y Plantas Medicinales (AFEPADI).

6. El convenio del sector del comercio de Cataluña para los años 2003-2004 lo firman las federaciones de comercio de CCOO y UGT y la patronal Confederación del Comercio de Cataluña.

7. El XIV Convenio de la Industria Química para el período 2004-2006 lo firman las federaciones sindicales pertinentes de CCOO (FITEQA) y de UGT (FIA) y la Federación Empresarial de la Industria Química Española (FEIQUE). 
Destaca, especialmente, el compromiso de configurar un "plan sectorial para promover la igualdad de oportunidades», en el que, a partir de un estudio de la situación de esa cuestión en la química, se arbitrarán medidas para hacer del sector uno de los más avanzados en el desarrollo de políticas de igualdad. Además de ese compromiso, el convenio recoge las regulaciones establecidas por la ley de conciliación, detallándolas de forma notoria para cada circunstancia (en el extenso capítulo VI del convenio sobre desplazamientos, dietas, licencias y excedencias) y ampliando en ocasiones el alcance de las medidas recogidas en la ley. Las mejoras más significativas se dan en las siguientes cuestiones:

- Regulación de la reducción de jornada por motivos familiares.

- Reserva de puesto de trabajo por maternidad.

- Excedencia por cuestiones familiares y licencias sin sueldo con motivo de la atención a familiares por enfermedad.

Aunque, sin duda, el convenio de química es el que dedica mayor atención a las cuestiones relacionadas con la conciliación, vale la pena subrayar que, en sus cláusulas, más que mejorar la legislación, lo que fundamentalmente hace es recogerla y concretarla para las empresas del sector. El convenio de comercio catalán es mucho más limitado y restrictivo, mientras que el de preparados alimenticios se sitúa en una posición intermedia. La situación contrapuesta de la química y del comercio es igualmente visible en las argumentaciones sobre la conciliación que realizan los responsables de las organizaciones empresariales catalanas que hemos entrevistado: Federación Empresarial Catalana del Sector Químico (FEDEQUIM) y Confederación del Comercio de Cataluña, organización que representa al conjunto de empresas del sector con la estricta excepción de cuatro de las mayores firmas del mismo. Brevemente expuestas, conviene resaltar algunas consideraciones que derivan de esas entrevistas, siendo de particular interés las que tienen que ver con las hipótesis que guían la investigación y que se abordan en un artículo anterior de este monográfico (Carrasquer, Martín Artiles y Massó, 2006) ${ }^{8}$.

De este modo, los entrevistados tienen un escaso conocimiento de las directivas europeas que dan pie a la Ley de conciliación y no creen que hayan tenido incidencia sobre la negociación colectiva. Mayor peso ha tenido la propia ley en el convenio de químicas, aunque, como señala el responsable entrevistado de la patronal sectorial, es sobre todo la insistencia sindical la que ha dado lugar a la inclusión de cláusulas relativas a la conciliación. De hecho, la conciliación tiene poca relevancia en el convenio de comercio, mientras que se le otorga más en el de químicas.

8. Respecto a las «buenas prácticas» en algunas empresas, sobre las que establecíamos la hipótesis de que podían actuar como ejemplos para otras, el responsable de la Confederación del Comercio de Cataluña señala el caso de Mercadona y el de FEDEQUIM el de Farmacia, aunque, en ambos casos, sin atribuirles especial relevancia y expresando que las peculiaridades de cada empresa hacen poco extensibles experiencias particulares de este tipo. 
[El tema de la vida laboral y familiar] son peticiones que están recogidas en la plataforma de los sindicatos, que plantean una serie de peticiones concretas en este bloque y se discuten [...] En el convenio de químicas ya había temas de conciliación. Lógicamente desde el 99, con la Ley, ha habido avances muy importantes porque [...] los sindicatos han querido incorporar esos temas en los convenios. (E12)

No, no hay en el convenio [cláusulas relativas a la conciliación]. Hay cosas en temas de bodas, necesidades familiares [...] Los sindicatos no lo han planteado. Hace poco hemos renovado el convenio y la conciliación no es un tema que se haya planteado. (E11)

El tema de la conciliación está más avanzado [en los convenios] que otros temas [...] ha habido una incorporación de la mujer al mercado de trabajo y también un incremento de la natalidad [...] por tanto es un tema que se ha de afrontar de manera seria y concreta. (E12)

Por otra parte, aunque se defiende una perspectiva de la conciliación válida para hombres y mujeres, en la práctica se alude a su mayor incidencia en la gestión de las políticas de personal relativas a éstas últimas. Esto es especialmente relevante, ya que tiene que ver con la propia concepción del significado de la conciliación y permite situar mejor la posición de las organizaciones empresariales al respecto.

No se discrimina en principio [...] hablamos tanto de la mujer como del hombre. Pero aunque no sea sólo para las mujeres, realmente las mujeres son las más afectadas nos guste o no [...]. En la conciliación se trata de adoptar las medidas adecuadas para que, sobre todo las mujeres que son las más afectadas por el tema doméstico, puedan compatibilizar un poco lo que es su trabajo con su obligación como madre o como lo que sea. Piensa que la vida familiar no es sólo el tema de los hijos, también puede ser el de la gente mayor, cada uno tiene su problemática. (E12)

En este mismo sentido, conviene subrayar que el responsable empresarial de la Confederación de Comercio de Cataluña entiende la conciliación desde las exigencias que el conjunto social efectúa sobre las empresas del sector en términos de horarios, con el fin de que los ciudadanos puedan efectuar sus compras sin que se lo imposibilite su desempeño laboral. Su discurso es muy elaborado, está ampliamente estructurado y muestra la preocupación de la organización que representa sobre la cuestión:

Creemos que los horarios comerciales son una falsa polémica cuando se plantea su liberalización [...] la actividad comercial es tan importante en nuestra sociedad [que sus horarios] no pueden organizarse sólo desde el punto de vista del consumidor. El efecto sobre el conjunto de la ciudadanía de una liberalización de horarios iría más allá de lo que es el consumo puro y duro [...] Somos un sector clave, un sector que preserva la cultura, las tradiciones, da vida a las ciudades, configura modelos de convivencia y, por tanto, aportamos muchos valores sociales [...] En el tema de la conciliación, en Europa, por la tradición europea, habría que reservar los domingos como el día prioritario de descan- 
so de todos los sectores productivos e intentar conseguir que al menos un día a la semana la gente, los ciudadanos europeos puedan encontrarse para mantener relaciones familiares, de amistad, etc. (E11)

El extracto anterior es un resumen mínimo de las argumentaciones extensamente desarrolladas en la entrevista. Esas argumentaciones, dan cuenta del interés de una organización empresarial en la defensa de los intereses de la mayor parte de sus representados, pequeñas y pequeñísimas empresas, imposibilitadas de hecho para abrir sus instalaciones en festivos como desearían algunas grandes firmas. Tan interesante como esa concepción de la conciliación es el hecho de que cuando se advierte de las dificultades en ese orden que pueden tener las personas empleadas en el sector, la conciliación desaparece como cuestión importante. De ahí, la poca relevancia que se le da en la negociación colectiva:

Claro, si lo miramos desde dentro, desde la gente que trabaja en un sector que está absolutamente sacrificado en beneficio del consumidor [...] ¿̨cuándo podrá ir a comprar el trabajador?, porque si todos cerramos a las ocho, ¿cuándo comprará la dependienta que tenemos? Pero resulta que no sé como se lo hacen, en el buen sentido, como se organizan, pero en las casas de nuestros trabajadores tienen productos y todos van bien vestidos y todos compran cotidianamente. Eso quiere decir que existen mecanismos propios, porque la gente trabaja cinco días y no seis o trabajaría más de cuarenta horas, quiere decir que hay planteamientos de rotación y alternativas del propio sector. (E11)

A tenor del entrevistado, no parece que haya, pues, problemas para las personas que trabajan en el sector, cuando es uno de los que mantiene horarios que dificultan en mayor medida la posibilidad de combinar la vida laboral, familiar y personal. Sin embargo, alguna de las reflexiones del responsable de la Confederación del Comercio catalán entronca con una idea de conciliación que consideramos particularmente importante, en tanto que va más allá de la interacción entre el trabajo y la familia y se extiende al conjunto de aspectos que componen los elementos vitales de la persona. Así, cuando se refiere a las dificultades de contratación de personal que experimenta el sector, indica que, al margen de los salarios, el problema es que los jóvenes no desean los horarios que se les marcan:

Hay gente que ni pagando más no viene porque prefiere tener el viernes libre o jornada intensiva todo el año [...] ¿Qué podemos hacer? Hemos de prestigiar la actividad, darle más valor social y buscar maneras de encontrar gente, estudiantes, personas de mayor edad, etc. Y hacer jornada a medida y cubrir lo que se cubría con un trabajador en ocho horas al día, a lo mejor con tres trabajadores que hagan turnos. Claro, eso hace que la organización y la mentalidad de la empresa también haya de cambiar. (E11)

Estas últimas reflexiones apuntan a la importancia de la empresa como el lugar primordial en que se concretan las dificultades para conciliar la vida fami- 
liar, laboral y personal (o los tiempos y recursos laborales con los que se configura la vida de las personas). De hecho, los dos responsables entrevistados subrayan la importancia de la gestión de la conciliación en el marco de la empresa, relegando la relevancia de la regulación sectorial de esta temática; aunque, especialmente en el sector de químicas, se considera que en el futuro será una cuestión que ganará en importancia. A continuación, y abundando en la concreción en el terreno de la empresa, explicitamos los principales resultados del estudio que hemos realizado sobre tres casos concretos.

\section{Las empresas punteras en conciliación}

Si bien el estudio de los referentes y las prácticas más innovadoras es un elemento capital para saber lo que acontece en las empresas españolas en materia de conciliación por su creciente y notable difusión en los principales círculos empresariales, los análisis al respecto son escasos y tienen un fuerte sesgo normativo de corte managerial. De hecho, ni siquiera nos encontramos con estudios que planteen los grandes modelos internacionales sobre esta temática con un cierto afán explicativo.

A este respecto, la literatura anglosajona, especialmente importante en este terreno por el desarrollo de esas políticas en empresas de los Estados Unidos y del Reino Unido, enfatiza la existencia de dos grandes marcos de actuación empresarial en el terreno de la conciliación con referentes, estrategias y prácticas concretas: el modelo conocido como family-friendly y el denominado work life balance (Baylin, Drago y Kochan, 2001; Rigby, 2004).

El primer modelo, etiquetado como family-friendly, está orientado a responder a las nuevas demandas sociales derivadas de los cambios demográficos y en el ámbito familiar, en especial las referidas a la atención de menores y de mayores dependientes, desde parámetros empresariales claros y homogéneos válidos para el conjunto de la plantilla y que establecen una norma ideal de empleado. Las estrategias ejemplares para su desarrollo son las respuestas empresariales proactivas que se entienden como satisfactorias para resolver problemas personales de la plantilla. Dichas estrategias apuntarían a facilitar una mayor implicación laboral a colectivos susceptibles de comportar importantes costes de rotación a las empresas por su notable capacidad de movilidad ascendente laboral, así como por realizar actividades de alta cualificación efectiva. En ámbitos con escasas diferencias respecto a la familia urbana tradicional regida por el modelo male breadwinner, afectaría especialmente al colectivo de mujeres. En menor medida, podrían responder a adecuar la norma ideal de empleado de la empresa a lo dictaminado por ámbitos externos a ella. En este caso, las directivas comunitarias y la legislación española, o lo propiamente acordado en la negociación colectiva, pueden ser imperativos poco costosos para las empresas, así como referentes de demandas de los empleados a resolver. Las principales prácticas en este orden son, sobre todo, los servicios facilitados por las empresas para atender a demandas familiares (guarderías, cheques para el servicio de atención domiciliaria, etc.) y, en menor medida, los 
permisos y las excedencias de paternidad y maternidad o de atención a ancianos dependientes. En este terreno, el trabajo a tiempo parcial sería también una práctica muy afín a la naturaleza de este modelo family-friendly, así como también lo serían medidas menos aplicadas, pero bastante difundidas por la literatura managerial, como la formación y las guías de orientación a los trabajadores para una mejor gestión personal de la conciliación entre vida familiar, personal y laboral.

De acuerdo con los autores citados arriba, éste sería el modelo empresarial más común. De hecho, es un modelo con bajos costes financieros y organizativos para la empresa, así como de menores oportunidades e incentivos inmediatos y de desarrollo de carrera profesional para los trabajadores. Con todo, los asalariados más susceptibles de ser objeto de estas medidas, los más cualificados con carreras profesionales establecidas, sí pueden experimentar costes importantes, en cuanto alguna de aquellas medidas puede comportar problemas para su promoción laboral, mientras que para los trabajadores menos cualificados y menos centrales para las empresas, disfrutar de estas acciones puede ser problemático si afectan al salario y a la estabilidad laboral. Así, para muchos autores, el desarrollo de una clara cultura empresarial al respecto y el que esas medidas no conlleven costes para los empleados a corto plazo (salarios, tiempo de trabajo pospuesto, etc.) o a medio plazo (estabilidad, promoción, etc.), serían los factores básicos para su potenciación (Baylin, Drago y Kochan, 2001; Rigby, 2004).

El segundo gran modelo se categoriza en términos de work life balance; en la doble acepción de balance en tanto que equilibrio no siempre fácilmente determinable ni asumible, dados los marcos, las ideas, las restricciones y los intereses de los actores implicados, y de work y life al integrar simétricamente aspectos diversos de la vida laboral, familiar y personal de los trabajadores. Este modelo responde a la insatisfacción de las empresas en torno a las formas y a la intensidad de la prestación laboral, así como a la insatisfacción de los trabajadores por sus pocas posibilidades de adecuar mejor sus actividades laborales con las familiares o personales. Es, por otro lado, una respuesta a ideas e intereses diversos sobre qué y cómo conciliar. Las respuestas empresariales ejemplares para su desarrollo son las que integran las medidas de conciliación en el marco de las políticas integrales de gestión de los recursos humanos. Así, las diferentes medidas de desarrollo de flexibilidad laboral cuantitativa y cualitativa externa e interna, que se redefinen considerando aspectos de conciliación y muchas veces de forma regulada con los empleados y los sindicatos, serían las estrategias más innovadoras y significativas. En líneas más generales, sería un modelo en cierta medida compatible con el paradigma de la flexsecurity (Wilthagen y Tros, 2004), al integrar aspectos diversos de las lógicas productivas y extramercantiles de los trabajadores y regulados colectivamente. En última instancia, obecederían, con todo, a la búsqueda empresarial de mayor implicación y productividad por parte de los trabajadores con la posibilidad de llegar a acuerdos de suma positiva entre las partes. Las prácticas más usadas serían aquellas relacionadas con la flexibilización del tiempo de trabajo, 
como los modelos de flexitime o de computación temporal a largo plazo de la prestación laboral, facilitadoras de mayor cantidad de tiempo productivo para las empresas y de más tiempo de uso personal para los empleados. En este orden de cosas, medidas como el teletrabajo (en realidad, más publicitada que implantada), así como las excedencias para la formación continua, serían acciones significativas.

De acuerdo con la literatura (Baylin, Drago y Kochan, 2001; Rigby, 2004), este sería un modelo menos difundido y de concreción más diversa, en función del contexto empresarial y ocupacional y del grado de carácter proactivo o reactivo de las medidas en términos de work life balance. En cualquier caso, en su desarrollo más común, es un modelo con dificultades de aplicación por los costos organizativos de rediseño y ajuste constante que puede comportar; así como por los costos a asumir para proporcionar incentivos a los empleados acordes a la satisfacción de sus demandas de conciliación. En general, en la práctica también se aplica más a trabajadores cualificados y centrales de las plantillas con mecanismos de regulación laboral individualizados. En estos casos, los costes se compensan por la naturaleza altamente cualificada de la prestación laboral y por responder a demandas específicas de colectivos que tienen buenas condiciones de empleo. De hecho, al igual que para el anterior modelo family-friendly, una cultura empresarial claramente definida es un factor de primer orden para su desarrollo, ya que para los empleados acogerse a estas medidas suele ser siempre costoso en lo que se refiere a sus perspectivas de desarrollar carreras muy profesionalizadas.

No ajeno a los planteamientos del modelo work life balance, la incorporación de la conciliación en el discurso de gestión de recursos humanos identifica frecuentemente conciliación y flexibilidad (Watson, 2004). Así, por ejemplo, empresa flexible es un término ya habitual para referirse también a firmas que introducen medidas de apoyo a la conciliación ${ }^{9}$. De ahí también que diversos estudios indiquen como las medidas de conciliación suelen chocar con las políticas y las culturas dominantes en las empresas, en particular con la idea de que la presencia en el trabajo y la disposición e intensificación laboral son señales de compromiso e interés con la empresa (De Luis y otros, 2003). Por ello, como apuntan Gómez y Martí (2004), la mayoría de las medidas que establecen las empresas en cuanto a flexibilidad y conciliación en sus políticas de recursos humanos se aplican poco en la realidad. Quizás porque, con frecuencia, la creciente intensificación del trabajo y la búsqueda empresarial de la buena disposición hacia ella por parte de los trabajadores sea incompatible con políticas efectivas de conciliación.

Por otra parte, algunas de las acciones empresariales asociadas con la conciliación remiten también, al menos como objetivo subsidiario, a la aparición

9. Con este término, los estudios de IESE (2004) en Barcelona se refieren a las medidas de conciliación de la vida laboral y familiar adoptadas por las empresas; o la creación de los Premios Empresa Flexible, concedidos a las empresas que destacan por su apoyo a la conciliación de la vida personal y profesional. 
pública de la entidad como una empresa «socialmente responsable» y que entiende que ese carácter debe ser ampliamente publicitado. En efecto, los estudios que enfatizan esas medidas lo hacen calificándolas con frecuencia de «buenas prácticas», una idea desarrollada desde los organismos de la UE y que estima que, para difundir en el tejido empresarial determinado tipo de políticas, cabe hacer conocer al conjunto social las iniciativas positivas en ese terreno emprendidas por algunas de ellas (IESE, 2004). De este modo, las empresas que las promueven se benefician de formas de reconocimiento diversas que sirven para mejorar su imagen corporativa entre sus clientes efectivos y potenciales y entre el conjunto social.

En la actualidad, hay diversas iniciativas que promueven y/o estudian la difusión de prácticas de conciliación, propias tanto del modelo family-friendly como del work life balance. Las iniciativas que finalmente se publicitan y se dan a conocer recogen la presencia de un limitado número de empresas en todas ellas. Así, por ejemplo, el Programa Óptima, al que se acoge la empresa Farmacia analizada en este artículo, promovido por el Instituto de la Mujer para favorecer la igualdad de oportunidades entre los dos sexos, impulsa estrategias que permitan compatibilizar las responsabilidades laborales y familiares. En el programa participan más de sesenta empresas, muchas de las cuales aparecen con frecuencia mencionadas por el desarrollo de «buenas prácticas» en términos de conciliación o de promoción de la igualdad entre hombres y mujeres en la empresa; aspectos éstos que, aunque no implican lo mismo, son frecuentemente identificados entre sí. Buena parte de las empresas con prácticas relevantes de conciliación, que cita el Informe Randstat (IEL, 2004) sobre calidad del trabajo, participan en el programa mencionado: Danone, El Pozo, Banco Santander-Central-Hispano o Mercadona, entre otras. Lo mismo ocurre con las medidas en pro de la conciliación recogidas por IESE (2004), que cita, entre otros, los casos de: IBM, Caja Madrid, Procter\&Gamble, Nestlé, Unión Fenosa, La Caixa, o Novartis, pertenecientes a sectores tradicionalmente masculinizados, pero donde la presencia de la mujer es creciente desde hace años ${ }^{10}$. Algunas de esas empresas, como es el caso del Banco Santander-CentralHispano o el de Repsol, han llegado a acuerdos con los sindicatos para desarrollar medidas vinculadas con la conciliación, acuerdos que no suelen ser habituales en las empresas que impulsan políticas de este tipo.

Aunque podríamos describir otros casos (Farmacia, uno de los que estudiamos, es de los más conocidos y publicitados), las empresas que desarrollan políticas de conciliación son poco numerosas (véase nota 1). De hecho, un estudio realizado para el Instituto de la Mujer (GPI Consultores, 2005) muestra que un $46 \%$ de la población en edad laboral opina que la conciliación es complicada o muy complicada y un 66\% de la misma pagaría más impuestos a cambio de una mejora en los recursos de apoyo. Según el mismo estudio, un

10. Para una descripción de las empresas más innovadoras, véase, además Cuenca (2005), Cruzado (2004) y El País Semanal (2005). 
66,9\% de responsables de 800 empresas encuestadas radicadas en España considera que la empresa privada debe tener una orientación exclusiva hacia el logro de la máxima productividad, un $61,6 \%$ estima que las dificultades de compaginación entre la vida laboral y la familiar deben ser solucionadas por las familias de forma privada y, además, un $41,3 \%$ de aquellos responsables empresariales afirma que la aplicación en la empresa de medidas de conciliación limita la competitividad. Asimismo, se advierte en ese mismo trabajo que las medidas no recogidas en la ley más aplicadas en las empresas son la flexibilidad en la hora de comienzo y finalización de la jornada (36\% de las empresas encuestadas) y la libre elección de turnos e intercambios de los mismos (31,6\% de los casos); mientras que la disposición de guarderías en el centro de trabajo, las ayudas económicas para el cuidado de personas dependientes y los permisos de paternidad más allá de lo que obliga la ley, se dan únicamente en el $1,4 \%$, el $2,8 \%$ y el $8 \%$ de empresas, respectivamente.

También son significativas al respecto las diferentes prácticas de conciliación de los empleados en función de su posición económica. Los más desfavorecidos, normalmente empleados poco cualificados o semicualificados y que ocupan las categorías profesionales inferiores, utilizan recursos internos de la familia (sobre todo la asistencia de la abuela materna para la atención de los hijos menores de edad), reconocen un mayor peso de la madre como responsable principal de la dispensación de cuidados familiares y una menor participación del padre y, asimismo, consideran que las necesidades de conciliación tienen una clara incidencia en los abandonos del mercado de trabajo. Los mejor ubicados en términos laborales, generalmente trabajadores cualificados, destacan por la elevada contratación de servicios de apoyo a la cobertura de sus necesidades familiares en el mercado, por una menor asimetría de género en el reparto de las tareas domésticas y por tener la conciliación una menor incidencia en los abandonos del mercado de trabajo (GPI Consultores, 2005).

De los estudios de casos realizados en centros de trabajo ubicados en Cataluña de las multinacionales Química, Alimentación-Comercio y Farmacia, nos encontramos con empresas de distinto perfil en función de los tipos ideales family-friendly y work life balance ${ }^{11}$. Las dos primeras se caracterizan por el desarrollo tradicional de políticas de recursos humanos racionalizadas y reguladas, condicionadas en parte por los dos convenios colectivos sectoriales de químicas (Química) y alimentación-comercio (Alimentación) analizados anteriormente. Igualmente, ambas vienen adoptando medidas afines a la conciliación desde hace bastantes años, en un marco de relaciones laborales regulados por un convenio para los diversos centros de trabajo de la empresa. Paralelamente, alimentación-comercio se caracteriza por gozar de cierta reputación en círculos empresariales en materia de conciliación, siendo una de las empresas del Programa Óptima del Instituto de la Mujer. Por su parte, la divi-

11. Para los estudios de caso de Química, Alimentación-Comercio y Farmacia, se realizaron entrevistas en profundidad a responsables del Departamento de Recursos Humanos y del Comité de Empresa de cada una de esas empresas. 
sión española de la farmacéutica de origen suizo Farmacia reviste el especial interés de ser una empresa que, con una política también sistematizada de recursos humanos y sujeta al mismo convenio colectivo que Química, elabora una iniciativa específica en materia de conciliación, el Proyecto T, que goza de gran predicamento en círculos empresariales. Analizaremos, seguidamente, como se concretan las políticas de conciliación en esos tres casos.

\section{A) Quimica}

De acuerdo con Recursos Humanos, Química históricamente ha tenido en cuenta la integridad personal de sus empleados. De ahí su lema corporativo "La calidad de vida es igual a la calidad de trabajo» (E15). En materia específica de conciliación, entiende que «la conciliación es un derecho individual definido por la legislación y la empresa no hace otra cosa que la aplicación de la misma» (E15). De hecho, en la actualidad se limita al cumplimiento de la ley y del convenio de químicas, aunque, cabe advertir, sigue aplicando medidas que estaban bastante desarrolladas con anterioridad. En esencia, hablamos de permisos de maternidad y lactancia, que afectan sobre todo a mujeres y, en menor medida, a permisos para la realización de estudios. Asimismo, la empresa retribuye parte de los costes de guardería de los hijos de sus empleados, tras dejar de facilitar el servicio de guardería ofertado desde los años setenta hasta inicios de los noventa por las escasas demandas, según se nos indica, de la plantilla.

Quizás en un futuro se desarrollen otras iniciativas, pero actualmente eso no es viable, por la prioridad de adaptar las diferentes divisiones de la multinacional a un amplio proceso de reestructuración que se encuentra en marcha en la actualidad. Con todo, es una empresa que facilita buenas retribuciones, condiciones de flexibilidad en los horarios de entrada y de salida y posibilidades de concentrar la jornada, lo que permite a los trabajadores acceder a servicios proporcionados por el mercado con recursos y tiempo suficiente para atender a sus necesidades (E15). Sin embargo, esas posibilidades se limitan esencialmente a los trabajadores de oficinas, de edades intermedias y con cierta presencia de mujeres, sin que las medidas se extiendan a las secciones de producción. Por otro lado, la empresa no se plantea cambios sobre esta cuestión a través de la negociación colectiva.

Esta lectura de las acciones vinculadas a la conciliación de la dirección de Química coincide parcialmente con la que proporciona CCOO, sindicato mayoritario del comité de empresa de las oficinas centrales. Si bien es una empresa paternalista y cumplidora con la legislación, con relativamente buena interlocución con los sindicatos y condiciones de empleo, la conciliación no es abordada de la mejor manera. Así:

Lo que nosotros entendemos por conciliación, y que estamos intentando aplicar, es conciliar nuestra vida individual, nuestra vida personal con la faena, en un momento en que la faena cada vez ocupa, parece, más tiempo, más espacio 
en nuestra vida, y es un poco buscar las reglas de juego para poder conciliar los dos ámbitos.[...] a parte de los cambios en la estructura social [...]. Por ejemplo, el tiempo que necesitan las mujeres preparto, que quiere decir marchar en horas de trabajo; estar fuera no sé cuantas semanas del parto; cuando vuelves, con el crío pequeño, el tiempo de lactancia y la reducción de jornada. Está claro, todo esto son reducciones del tiempo de trabajo en un momento en el que hay carga, presión de la faena, exigencia de resultados. Entonces esto lo hemos vivido como problemas, es decir, ha habido gente que ha tenido pressing con esto. [...] Más con los jefes que con los compañeros. (E16)

De hecho, la conciliación tiene cada vez más importancia para los trabajadores de la empresa, por la mayor intensificación del trabajo y el ajuste de la plantilla. Asimismo, afecta más negativamente a las mujeres, al vincularse los costes de coordinación que implica la conciliación a sus cuestiones personales, y, desde ahí, repercute negativamente sobre sus condiciones y posibilidades de empleo. CCOO considera, además, que, a pesar de disponer de flexibilidad en los horarios de entrada y de salida del trabajo y de salarios relativamente altos, la atención y el cuidado de los hijos menores de edad y, más aún, de las personas de edad avanzada, son los aspectos más problemáticos para la conciliación y resultan muy difíciles de abordar por parte de los trabajadores (E16).

El sindicato contempla, para el próximo convenio general de Química, abordar la conciliación. Bajo el marco de un documento bastante elaborado, plantean crear una comisión paritaria que impulse mejoras de igualdad de género y que, específicamente, se fomenten los contratos a tiempo parcial a través de puestos de trabajo compartidos, para propiciar la atención y el cuidado de las personas mayores y la realización de estudios. Estas cuestiones afectarían especialmente a los hombres y a las mujeres de oficinas, y no tanto a producción, donde hay trabajo por turnos. Es, con todo, un tema de difícil concreción en el ámbito específico que se plantea, impulsar el puesto de trabajo compartido, por el menor salario y computación para las pensiones de jubilación que implica un contrato a tiempo parcial (E16).

\section{B) Alimentación-Comercio}

De acuerdo con Recursos Humanos, Alimentación-Comercio ha implementado tradicionalmente un conjunto de iniciativas vinculadas a la conciliación. Hasta 1997, con el impulso de un servicio de guardería, que ya no se facilita por la escasa demanda de la plantilla, y posteriormente con la cofinanciación de este servicio a sus empleados. A ello se une la flexibilidad de entrada (entre las 7.30 y las 9.15) y de salida (de 17 a 19 horas) y la concentración de jornada los viernes y vigilias de fiestas, medida ésta implementada hace veintiocho años; así como la ubicación de servicios de banca, viajes y seguros en la empresa. Asimismo, se facilita la carrera profesional de las mujeres, evitando desplazamientos largos a centros de trabajo lejanos al domicilio para favorecer sus posibilidades de promoción. Desde 1998, Alimentación-Comercio se inscribe en 
el Programa Óptima, desarrollando medidas de igualdad de género, entre las que destacan evitar el lenguaje sexista para tipificar las categorías profesionales y ahondar en medidas que faciliten el desarrollo de la carrera profesional de las mujeres (E13). Con posterioridad, se ha promovido la reducción de jornada para empleados con hijos menores de seis años, se fomenta el empleo a tiempo parcial para los empleados del ámbito de producción y se exime a las mujeres de producción con hijos menores de un año de trabajar en el turno de noche.

Las medidas de conciliación son ya clásicas en la empresa. Su adscripción al Programa Óptima, como que parte de ellas sean recogidas en el convenio colectivo de sus centros de trabajo, obedece a mejorar su racionalización. La valoración social de esa política es del todo positiva, como así lo aseveran los diversos premios y reconocimientos obtenidos. Por contra, Recursos Humanos considera que la Ley de conciliación no ha tenido efectos significativos, al tratar aspectos ya contemplados por la empresa (E13). En última instancia, sus acciones son un buen ejemplo de iniciativas que responden a criterios propios sobre esta cuestión, al tiempo que derivan sobre todo de la discrecionalidad empresarial, más que de responder a los criterios fijados por la legislación o por la negociación colectiva sectorial o de empresa. En la actualidad, Alimentación-Comercio trabaja en difundir las medidas adoptadas a través de la intranet de la empresa, sin plantearse acciones adicionales.

Por el contrario, desde el sindicato CCOO, representado en el comité de empresa, el fomento de la igualdad de género y la conciliación se entienden como acciones relativamente clásicas de la empresa, pero con carácter reactivo y de escaso alcance. Obedecen sobre todo a conflictos previos y a la necesidad, por parte de la empresa, de mejorar su imagen (E14). Es precisamente a raíz de un conflicto por una denuncia relacionada con este tema en el año 1997, cuando la empresa se acoge al Programa Óptima para contrarrestar la mala imagen que había adquirido después del conflicto. Para CCOO, la Ley de conciliación sí ha sido fundamental. Sólo basta mencionar que, antes de la ley, muchas mujeres no agotaban la baja de maternidad por presiones de los mandos y, en última instancia, para hacer méritos en la empresa y tener posibilidades de promocionar. Por su parte, también ha sido importante para reclamar derechos adquiridos, como es el caso de la reclamación de compensaciones por el servicio de guardería cerrado en 1996, reconocidas en la actualidad por una sentencia del Tribunal Superior de Cataluña que fue recurrida por la empresa en el Tribunal Constitucional. En general, sin embargo, las acciones en torno a la conciliación siguen siendo de mínimos, selectivas e individualizadas (E14).

En este último sentido, como indica $\mathrm{CCOO}$, la lectura de la conciliación por parte de la empresa está destinada y aplicada de forma casi exclusiva a directivos, técnicos, comerciales y administrativos de los servicios centrales. Sus medidas se entienden, además, como iniciativas de potestad empresarial. Más concretamente, la conciliación que promueve la empresa es que el personal comercial y ejecutivo femenino no tenga que hacer traslados largos desde 
el domicilio hasta el centro de trabajo, ubicando a esas personas en los centros más próximos a su domicilio, para así poder hacer compatible la vida familiar y laboral ${ }^{12}$. Para el conjunto de los empleados, lo más valorado es la flexibilidad en el horario de entrada y de salida, la concentración de jornada los viernes (se suele acabar a las 15.00) y la propia mejora de los permisos que ya contempla la ley. El fomento de la igualdad de oportunidades en las condiciones de empleo contemplado en el Programa Óptima tiene, según el responsable sindical, una nula incidencia (E14). La empresa no sigue este criterio para la contratación, ni para la formación o los mecanismos de promoción. Lo único que ha cambiado es que se presentan más mujeres a los procesos de selección, generalmente licenciadas, y eso conlleva una cierta paridad.

Como puede verse, el abordaje de la conciliación reproduce políticas de tipo paternalista por parte de la empresa. De hecho, Alimentación-Comercio España no tiene un plan de igualdad de oportunidades negociado y, en los diversos convenios colectivos de los centros de trabajo, las mejoras para la conciliación se limitan a: a) la jornada laboral flexible en la entrada y la salida y concentración de jornada los viernes; $b$ ) un día más de permiso que en la legislación para atender fallecimiento, accidentes o enfermedad grave u hospitalización del cónyuge, hijos, padre o madre; $c$ ) ayuda económica a guardería para los hijos menores de cinco años, y d) reducción de la jornada en una hora para los casos de guarda legal de un menor de seis años y de discapacitados. En este terreno, lo más significativo para el futuro inmediato será el impulso de lo recogido en el convenio colectivo sobre la legislación vigente (E14).

\section{C) Farmacia}

Farmacia, por su parte, desarrolla desde hace un año y medio el Proyecto T, dirigido exclusivamente a la conciliación ${ }^{13}$. De acuerdo con Recursos Humanos, está extendido en toda la multinacional, pero hay diseños concretos para cada división. En su formulación, rompe con la tradicional lectura de la conciliación como derivada de las nuevas necesidades familiares para entenderla como un problema "personal, social y familiar» vinculado con empleos con tareas por objetivos, de alto rendimiento y dedicación temporal y que se han de afrontar primando una mejor gestión del tiempo. Como se nos indica, sus premisas difieren del modelo familista y se fundamentan en:

12. Los beneficios de esa acción son, en cualquier caso, limitados, ya que la empresa puede facilitar la elección entre unos pocos centros de trabajo a sus empleadas. Otras empresas también han implantado acciones similares, pero pueden alcanzar mayor calado cuando así lo facilita su localización territorial. Es el caso, por ejemplo, de Caja Madrid (IESE, 2004). Esta empresa procura ublicar a las mujeres que emplea y que tienen cargas familiares en centros cercanos a su domicilio. La profusión de oficinas bancarias en el territorio facilita la eficacia de aquella acción en mayor medida que en el caso de Alimentación-Comercio.

13. A efectos de mantener la confidencialidad de las empresas, el proyecto de esta empresa se etiqueta como Proyecto T. 
La empresa se ha dado cuenta que vale la pena favorecer y facilitar la vida de las personas en temas que podían ayudar en estas tres áreas (personal, social y familiar), entendiendo que no podemos añadir más tiempo a nuestras vidas, pero sí podemos hacerlas de mayor calidad, hacerlas más eficientes; por ejemplo: una de nuestras acciones es eliminar la burocracia, la gente aquí está trabajando muchas horas, funciona por objetivos, no como la Administración pública que estás de tal a tal hora y haces lo que te parece, tienes unos objetivos básicos, vas haciendo pero básicamente lo que has de cumplir es un horario. Aquí, en cambio, hay un horario establecido, pero aquí lo que hay básicamente son unos objetivos y la gente a lo que va es a cumplir los objetivos, si estas personas son capaces de cumplirlos con menos tiempo, perfecto, y esto la empresa lo facilita en todos los aspectos. Ésta es un poco la teoría que hay debajo de lo que es Proyecto T. (E17)

\section{El proyecto se basa en cuatro grandes pilares:}

1. Aumentar la eficiencia y eliminar la burocracia. Con las siguientes acciones: no realizar reuniones durante la primera hora de jornada laboral ni tampoco más tarde de las cinco (hora de finalización de la jornada); tipificar los correos electrónicos en función de su importancia y urgencia, y elaborar la base de datos Deja de hacerlo, simplifica a partir de reuniones habituales con los empleados.

2. Mentalidad positiva y equilibrada de los empleados. La acción más destacada en este sentido es la posibilidad de trabajar con vestuario informal los viernes.

3. Mejora del entorno laboral a nivel social y de la comunidad. Acciones de work life balance. Comprende actuaciones como: curso Desconexión, con la finalidad de evitar el estrés; curso Liderando reuniones efectivas, y el Club Proyecto T: acciones para mejorar las relaciones sociales entre los trabajadores, entre las que destacan facilitar prendas para actividades deportivas, descuentos en las entradas para algún evento cultural, organización de excursiones y el alquiler ocasional de un cine para el pase de películas dirigidas a los hijos de los empleados.

4. Recursos y servicios al colaborador. Supone: servicios de una empresa para asistencia sanitaria y atención a los pequeños y mayores con descuento y rapidez en la prestación; servicio de cátering del menú de la empresa del mediodía para la cena en casa a precios reducidos; concesión de coches familiares a los comerciales, y servicios de yoga y de masajes.

A tenor de lo expuesto por Recursos Humanos, lo que se ofrece son servicios opcionales para los empleados, nunca obligados. Están dirigidos especialmente a trabajadores de oficinas por la naturaleza de su trabajo, sin que se haya requerido su participación en el diseño ni en la implementación de tales servicios. De hecho, el proyecto no se ha difundido de forma integral a la plantilla, sino dando a conocer las acciones puntuales. Aunque la empresa desconoce el número total de trabajadores atendidos por el proyecto, su valoración 
es positiva, fundamentalmente, en tanto que las medidas que contempla sirven para mejorar la satisfacción laboral, aunque, como contrapartida, son notablemente caras: "A la empresa le es muy caro, cada curso de estos vale una burrada, mil euros un curso de cuatro horas, si haces veinte cursos, o las entradas de algún evento cultural o cerrar el Coliseum o la misma web vale una burrada. En suma, todo el coste es muy caro, esperemos que los beneficios sean que la gente realmente lo aproveche» (E17). En un futuro inmediato, se fomentará el teletrabajo, entendido como la medida estrella del work life balan$c e$, para trabajadores con necesidades personales muy concretas (se cita en concreto a las mujeres con maternidad reciente), aunque por el momento no se ha concretado ningún puesto de trabajo con ese perfil.

De acuerdo con Recursos Humanos, cuestión aparte del proyecto es la aplicación y cierta mejora de lo establecido por la Ley de conciliación. En este terreno, la empresa señala que mejora la ley en los siguientes puntos: ampliación de dos semanas más por baja maternal; equiparación de las parejas de hecho a los matrimonios, y equiparación de la adopción a los derechos de tener un hijo. Al igual que el conjunto del Proyecto T, se trata de iniciativas valoradas positivamente por los sindicatos, aunque, en la práctica, están igualmente recogidas en el convenio de la industria química.

Así, el presidente del comité de empresa del centro de trabajo de oficinas centrales y miembro de CCOO valora positivamente las iniciativas emprendidas, pero de forma muy negativa, su falta de negociación. De hecho, la empresa no tiene convenio propio y las relaciones laborales se rigen por un pacto de empresa firmado en 1998 que la dirección no ha querido renegociar. Valora también críticamente la escasa aplicación del Proyecto $T$ en todos los departamentos y el grado de discreción que tienen los directores de los mismos en su difusión (E18). En este sentido, la naturaleza de un trabajo por objetivos sujeto a remuneraciones por pluses, que implica un notable rendimiento y largas jornadas de trabajo y es el principal indicador de una promoción a discreción de los directores, los cuales, a su vez, están sometidos a unas relaciones similares con la gerencia, dificulta mucho el desarrollo del proyecto, especialmente su medida más interesante de hacer cumplir los horarios de trabajo y no programar reuniones después de las cinco de la tarde. Las otras medidas, por su parte, si bien pueden servir para mejorar el clima de trabajo, son bastante anecdóticas (E18). En otro orden de cosas, hay que subrayar que se trata de una acción que afecta exclusivamente a oficinas, siendo su principal vía de difusión la intranet de la empresa, una herramienta que no está a disposición de los empleados de producción.

\section{Conclusiones}

El análisis cualitativo que fundamenta este artículo sobre la definición y articulación de los intereses empresariales y sus prácticas más novedosas en materia de conciliación corrobora, como apuntan otros estudios, la escasa presencia de iniciativas proactivas en este terreno. Paralelamente, muestra la adecuación 
de las políticas empresariales de conciliación a una legislación familista y orientada a incidir, sobre todo, en la inserción y en la permanencia de las mujeres en el mercado de trabajo. En cualquier caso, algunas prácticas empresariales apuntan a reforzar la tesis según la cual la conciliación incide de forma novedosa durante los últimos años en la realidad de las empresas, pero entendiéndola desde ellas como un fenómeno de naturaleza externa y observándola más como un coste que como un recurso para configurar las políticas de gestión del personal.

De hecho, tanto de las valoraciones generales de las organizaciones empresariales confederales como de aquéllas referidas a la negociación de convenios significativos por parte de las organizaciones sectoriales, se puede postular una mera adecuación a los criterios mínimos establecidos por la Ley de conciliación. Una adecuación fuertemente condicionada por las iniciativas sindicales en los convenios más desarrollados, como ocurre en el de químicas, y, en términos discursivos, muy afín al modelo family-friendly anglosajón en su concreción más limitada. En otras palabras, la conciliación, desde la óptica del empresariado, se asocia a respuestas de naturaleza bastante coyuntural, delimitadas esencialmente por la ley y ante problemas familiares del personal que se entienden como responsabilidad básicamente de las mujeres. No es extraño, así, que, desde las organizaciones empresariales, se defienda también en este terreno la empresarización e individualización de las relaciones laborales a la hora de implementar acciones de conciliación; entendiendo, además, que la conciliación es un fenómeno de origen exógeno a la empresa y difícil de atender en lo que se refiere a las necesidades y a las demandas específicas del personal.

De los datos obtenidos en las entrevistas para la realidad concreta de empresas entendidas como innovadoras, es difícil establecer interpretaciones claras sobre la naturaleza y los efectos de estas acciones y, menos aún, inferir ideas para el conjunto empresarial español que elabora políticas de esta naturaleza. Con todo, sí que destacan un conjunto de aspectos que cabe subrayar para analizar la dimensión más proactiva de las iniciativas en este terreno. El primero de ellos es la inexistencia de una cultura empresarial que asuma claramente la necesidad de fomentar las medidas de conciliación, al tiempo que, cuando existen, se configuran en buena medida al margen de las estrategias que orientan la gestión de los recursos humanos. Así, Química tiene una posición notablemente reactiva y adaptativa ante el cumplimiento de lo exigido por la ley, a la vez que hace mención al Proyecto T de Farmacia como un claro referente de actuación, sin que contemple ninguna medida futura en esta línea. Alimentación-Comercio presenta, además de este perfil, un estilo de actuación bastante condicionado por las demandas sindicales y una voluntad de mejorar su imagen corporativa más que de impulsar acciones efectivas de igualdad de oportunidades. Farmacia, finalmente, parecería presentar una línea de actuación muy innovadora, pero sus acciones en términos de work life balance son poco más que aparentes, desconsiderando la gestión de la diversidad de demandas que los trabajadores pudieran expresar en este senti- 
do y, lo que es más singular, sin vincularlas a medidas clásicas a lo familyfriendly que permitan responder a las necesidades familiares más comunes de sus empleados.

En última instancia, nos encontramos con unas políticas de corte paternalista, selectivas, individualizadas y claramente productivistas. Así, un modelo como el de Alimentación-Comercio, reputado en diversos círculos empresariales, que podría adecuarse en cierta medida al modelo family-friendly, adolece de la concreción de una norma ideal de empleo para atender a las nuevas demandas familiares y personales de los empleados, como así se constata de los muy diferentes diagnósticos, valoraciones y expectativas que tienen al respecto Recursos Humanos y el sindicato CCOO. Es, asimismo, un modelo esencialmente dirigido a los colectivos centrales de la plantilla y que no es susceptible de negociación en el convenio colectivo. Por otro lado, del discurso empresarial sobre las acciones emprendidas, se puede inferir que conciliar es un asunto de mujeres y que sus medidas buscan facilitar el cumplimiento de sus obligaciones laborales. Más que un modelo de empresa conciliadora "amiga de la familia», parece que nos encontramos con un modelo de «mujeres con familia amigas de la empresa». Un modelo que define y subsume sus medidas de conciliación a cánones y orientaciones de corte productivista.

Farmacia, en teoría y en principio, parece desarrollar un modelo muy novedoso de work life balance. Pero todo apunta a que, en la práctica, sus acciones persiguen, sobre todo, fomentar un mayor rendimiento y disposición temporal por parte de los empleados, sin pretender que participen en el diseño de las mismas. A este respecto, es significativo que una medida como la acotación del tiempo de trabajo a lo que establece la jornada laboral y la realización de reuniones únicamente durante esa jornada, se presente como una acción novedosa y, según la propia dirección de Recursos Humanos, aún en fase de consecución. De hecho, según la literatura especializada, las medidas emprendidas serían más propias del modelo family-friendly en sus versiones menos comunes y poco innovadoras. Finalmente, no deja de ser chocante que el Proyecto T, ahora iniciado y que se supone importante para aumentar en un futuro inmediato la productividad laboral, se considere muy caro para la empresa y que no hayan estimaciones claras sobre su actual incidencia entre la plantilla. De forma paradójica, el modelo de Química, más tradicional, establece una conciliación efectiva en términos de mínimos y relativamente acorde con los objetivos empresariales y sindicales. Es significativo al respecto que CCOO plantee, como medida posibilista en la futura negociación colectiva con la empresa, la contratación a tiempo parcial para puestos de trabajo compartidos y para facilitar la atención cotidiana de menores y de ancianos. Una propuesta que, si bien no se aleja de los modelos anglosajones de family-friendly, podría abrir una vía de actuación bastante innovadora si se concretara en la configuración de puestos de trabajo efectivamente compartidos en base a la conciliación de las actividades laborales, familiares y personales de la plantilla. 
En cualquier caso, la reticencia observada en la dirección de las tres empresas analizadas para que las cuestiones sobre la conciliación se negocien de manera formal con los representantes de los trabajadores o, incluso, de forma individual con la parte de la plantilla afectada, es una de las limitaciones más serias que hemos observado en las políticas de conciliación que impulsan las empresas. Al depender esas políticas de la discrecionalidad empresarial, su concreción y sus posibilidades de expansión futuras son extremadamente aleatorias y se vinculan poco con las necesidades de las personas para conciliar sus actividades laborales, familiares y personales.

\section{Entrevistas referenciadas}

Asociaciones empresariales

E11. Fomento Nacional de Cataluña. Federación Comercio.

E12. Fomento Nacional de Cataluña. Federación FEDEQUIM.

\section{Empresas}

E13. Dirección de Recursos Humanos multinacional comercio-alimentación.

E14. Presidenta Comité Empresa multinacional comercio-alimentación.

E15. Dirección Recursos Humanos multinacional químicas.

E16. Responsable Sindicato multinacional químicas.

E17. Dirección Recursos Humanos multinacional farmacéutica.

E18. Responsable Comité de Empresa multinacional farmacéutica.

\section{Bibliografía}

Acuerdo Interconfederal para la Negociación Colectiva de 2005. Madrid, 4 de marzo de 2005.

AlCÁZAR, M.A.; Murga, A. (2003). «Flexibilidad y relaciones laborales». En: Benito, C. de; DurÁn, F. (coords.). Relaciones laborales. Asignaturas pendientes. Madrid: AEDIPE.

ARgüelles, A.R.; MartíneZ, C.; MenÉNDeZ, P. (2004). Igualdad de oportunidades y responsabilidades familiares: Estudio de los derechos y medidas para la conciliación de la vida laboral y familiar en la negociación colectiva (2000, 2001, 2002). Madrid: Consejo Económico y Social.

Bailyn, L.; Drago, R.; Kochan, T. (2001). Integrating Work and Family Life: A Holistic Approach. Cambridge (Massachusets): MIT Sloan School of Management.

CAPITAL HUMANO (2001). «La conciliación de la vida personal y profesional modifica las culturas corporativas». Capital Humano, núm. 149

CARRASQUer, P.; MarTín ARTILES, A.; MASSÓ, M. (2006). «Discursos y estrategias en torno a la conciliación de la vida laboral y familiar en la negociación colectiva». Papers. Revista de Sociologia, núm. 83: 13-36.

CASADO, M.P. (2002). «Nueva tendencia en los servicios al empleado: equilibrio trabajo y vida». Capital humano, núm. 160.

CASALS, P. (2005). «Conciliació laboral i familiar». La revista online del CTESC, núm. 23, junio. Barcelona: Consell de Treball, Econòmic i Social de Cataluña. 
CEOE (2002). «Balance de la encuesta de CEOE sobre negociación colectiva 2001. Estructura y contenido de los convenios colectivos en España». Informes y Estudios. CEOE, núm. 88, Madrid.

- (2004). «Balance de la encuesta de CEOE sobre negociación colectiva 2003. Estructura y contenido de los convenios colectivos en España. Informes y Estudios. CEOE, núm. 97, Madrid.

CES (2003). La negociación colectiva como mecanismo de promoción de la igualdad entre hombres y mujeres. CES Informes 2/2003. Madrid: Consejo Económico y Social.

- (2004). Panorama sociolaboral de la mujer en España, núm. 38. Conciliación de trabajo y vida familiar: licencias parentales. Madrid: Área de Estudios y Análisis del Consejo Económico y Social.

Chinchilla, N. (2004). Indice de Empresas Familiarmente Responsables. Pamplona: IESE.

Chinchilla, N.; Poelmans, S. (2003). Dues professions i una família. Estudi sobre les families amb dos ingressos a Catalunya. Barcelona: Departament de Benestar i Família de la Generalitat de Catalunya.

CREADE (2001). Work and Life Balance. Barcelona: Pharma Consults.

CRUZADO, M. (2004). «Las empresas se enfrentan al reto de implantar políticas que equilibren vida profesional y personal». Capital Humano, núm. 176.

Cuenca, N. (2005). «Conciliar para vivir mejor». Muface, núm. 198. Madrid: Ministerio de Administración Pública.

El Pais Semanal (2005), núm. 1.490, 17 de abril. Madrid: El País.

EUROPEAN INDUSTRIal RELATIONS ObSERVATORY (EIRO) (2002). Reconciliation of work and family life and collective bargaining. Dublin: European Foundation for The Improvement of Working and Living Conditions.

GÓMEZ, S.; MARTí, C. (2004). La incorporación de la mujer al mercado laboral: Implicaciones personales, familiares y profesionales, y medidas estructurales de conciliación trabajo-familia. Navarra: IESE. Documento de Investigación, 557.

GPI CONSULTORES (2005). Estudio sobre la conciliación de la vida familiar y la vida laboral: situación actual, necesidades y demandas. Madrid: Instituto de la Mujer.

IESE (2004). Guía de buenas prácticas de la empresa flexible. Madrid: Comunidad de Madrid.

Instituto de Estudios Laborales (IEL) (2004). Informe Randstad. Calidad del Trabajo en la Europa de los Quince. Madrid: ESADE.

LÓPEZ, M.T.; VALIÑO, A. (2004). Conciliación familiar y laboral en la UE. Madrid: Consejo Económico y Social.

Luis, M.P. de; MarTínez, A.; Vela, M.J.; Pérez, M. (2003). «La implementación de prácticas flexibles para la conciliación de la vida familiar y laboral». Capital Humano, núm. 166.

Luis, M.P. de; Martínez, A.; Pérez, M.; Vela, M.J. (2004). «El conflicto trabajofamilia: un estudio empírico de factores familiares y laborales». Capital Humano, núm. 175.

RigbY, M. (2004). Equilibrio entre trabajo y vida no laboral: ¿Un concepto útil? Ponencia presentada en el Seminario "L'organització social del temps: conciliació de la vida laboral i familiar», organizado por el Grupo de Estudios Sociológicos sobre la Vida Cotidiana y el Trabajo (QUIT). Departamento de Sociología de la Universidad Autónoma de Barcelona. Bellaterra, 30 de enero. 
TORNs, T. (2005). «De la imposible conciliación a los permanentes malos arreglos». Cuadernos de Relaciones Laborales, núm. 23. Madrid: Escuela de Relaciones Laborales de la Universidad Complutense de Madrid.

WATSON, T. J. (2004). «HRM and Critical Science Analysis». Journal of Management Studies, vol. 41, núm. 3.

Wilthagen, T.; Tros, F. (2004). «The concept of "flexsecurity". A new approach to regulating employment and labour markets». Transfer, núm. 2/2004, Bruselas: ETUI. 